\title{
Dynamic Scattering from Semiflexible Polymers
}

\author{
Emmanuel Farge and Anthony C. Maggs* \\ Laboratoire de Physico-Chimie Théorique, ESPCI, 10 rue Vauquelin, \\ F-75231 Paris Cedex 05, France \\ Received October 5, 1992; Revised Manuscript Received May 6, 1993
}

\begin{abstract}
We discuss the dynamics of dilute and semidilute solutions of semirigid chains. The results can be applied to analyze recent light scattering experiments from solutions of actin filaments where an anomalous scaling of the dynamic structure factor was found. This anomalous scaling is explained as being due to anisotropic scaling of characteristic length scales parallel and perpendicular to a filament and leads to characteristic frequencies in the system scaling as $q^{8 / 3} \log (q)$.
\end{abstract}

\section{Introduction}

Dynamic scattering experiments have played an important role in elucidating the internal dynamics of flexible polymers. ${ }^{1,2}$ Many observed phenomena are now understood in terms of the scaling behavior of scattering functions. $^{3}$ The theory of the dynamics of semiflexible polymers is, however, much less developed; in this paper we analyze the case of solutions of semiflexible molecules; in particular, we analyze recent experiments on actin filaments. For this system the scattering dynamics are dominated by transverse bending fluctuations which we model with a simple Langevin equation.

Actin is a globular protein with a mass of 43000 daltons with a diameter of $3.5 \mathrm{~nm}$, which plays an important role in many processes in the living cell. ${ }^{4}$ It is present in large quantities in many cells where it has important structural and locomotive roles. The protein exists in two major forms: G-actin, a dilute solution of unassociated molecules, and F-actin, an aggregated filamentary structure. It is a protein which is easily purified and forms a model system whose physical properties have been studied in detail under highly idealized conditions. A filament consists of an assembly of actin molecules in a double helix structure with a pitch of $72 \mathrm{~nm}$. The molecules assemble in the presence of ATP via a polar growth mechanism to form stable objects with lengths of many tens of microns and diameter $a$ of the order of $7 \mathrm{~nm}$. These aggregated filaments contain bound ADP which stabilizes the structure and thus undergo only slow depolymerization under normal conditions. Thus, once polymerized, an individual filament can be considered as a stable object on the time scales important for dynamic light scattering experiments.

The configuration freedom and fluctuations of actin filaments have been studied with a number of techniques including electron microscopy, ${ }^{5}$ fluorescence microscopy, ${ }^{6,7,8}$ and dynamic light scattering. ${ }^{9,10}$ Information on the internal dynamics of filaments can also be extracted from detailed rheological measurements.11,12 Electron microscopy permits one to study single configurations of a single chain, but it is difficult to obtain extensive statistics for quantitative measurements of elastic constants. Fluorescence studies permit one to study the evolution of single-chain segments but involve the adsorption of a dye which could change the structure of the filament and hence its mechanical properties. Recently, ${ }^{13}$ detailed quasielastic light scattering experiments have been performed on dilute and semidilute solutions of actin filaments, and the results have been analyzed using ideas of dynamic scaling in polymer solutions. A particularly striking result was that over a large range of times and concentrations the intensity-intensity correlation function was shown to be a universal function of only the combination $q^{2.7} t$, rather that the two independent variables $t$ and $q$. This result should be contrasted with that found in flexible polymers where the correlation function depends on the combination $t q^{3}$ (Zimm model) or $t q^{4}$ (Rouse model). ${ }^{3}$ For the longest times considered in the experiments, interactions between filaments become important and the scattering form changes.

The major result of this paper is that on the time scales important for the experiment the dynamic structure factor $g(q, t)$ is a function of the combination $t q^{8 / 3}$ with, however, important logarithmic corrections. Rather surprisingly, we find that the system does not exhibit exact scaling; however, the effects which destroy scaling turn out to be weak. In section II of this paper we shall give a qualitative argument which leads simply to these results. The argument can be turned into an exact calculation, which is presented in section III. In the fourth section of this paper we shall give arguments that the dynamic structure factor $g(q, t) \sim \exp \left(-q^{2} t^{1 / 4}\right)$ for the longest times in experiments when interactions between filaments become important. Our theory gives estimates for the bending constant of a filament which are consistent with those found in microscopy.

\section{Scaling Arguments}

In this section we shall present some simple scaling arguments to describe dynamic light scattering from individual actin filaments. Microscopy shows ${ }^{6,7,8}$ that F-actin is a semiflexible chain with a persistence length $\xi$ of some $6 \mu \mathrm{m}$; thus for scattering vectors important in optical experiments we can locally describe the shape of the filament in terms of fluctuations about a straight rod. If the mean position of the filament lies along the $x$ axis, we shall describe the shape of the filament in terms of two independent fluctuations $\mathbf{r}(s)=\left(r_{y}(s), r_{z}(s)\right)$. For simplicity, let us consider the scattering from a single filament with the scattering vector $q$ perpendicular to the mean axis of the filament, $q=q \hat{\mathbf{z}}$. Such a description neglects all compressional and torsional degrees of freedom for a chain and is clearly only valid for phenomena occurring on length scales small compared to $\xi$.

Dynamic light scattering experiments measure the dynamic structure factor

$$
g(\mathbf{q}, t)=\frac{1}{N}\left\langle\sum_{n, m} \exp \left[i \mathbf{q} \cdot\left(\mathbf{r}_{n}(t)-\mathbf{r}_{m}(0)\right)\right]\right\rangle
$$

where the sum is over all molecules in the filament at positions $\mathbf{r}_{m}$. The decay of this correlation function is due to the progressive dephasing of the signal as a function of time; for this event to occur, a given protein molecule in 
the filament should move a distance of order $1 / q$. For an actin filament we can estimate the characteristic time for this process as follows:

The bending elastic energy associated with a deformation of the filament is given by ${ }^{14}$

$$
E=\frac{\kappa}{2} \int\left(\nabla^{2} \mathbf{r}\right)^{2} \mathrm{~d} s=\frac{\kappa}{2} \int p^{4} \mathbf{r}_{p} \mathbf{r}_{-p} \frac{\mathrm{d} p}{2 \pi}
$$

where $\alpha$ is the bending elastic constant which is directly related to the persistence length, $\xi=(2 / 3)_{\kappa} / k_{B} T$. The typical transverse fluctuations associated with a segment of F-actin of length $L$ can be estimated as

$$
\left\langle r^{2}\right\rangle \sim \frac{k_{B} T}{\kappa} \int_{1 / L}^{\infty} \frac{1}{p^{4}} \mathrm{~d} p
$$

where $T$ is the temperature. Thus we find a natural relation between length scales parallel, $L$, and perpendicular, $l$, to a filament: $l^{2}=k_{B} T L^{3} / \kappa$. Similarly, we find the relation between wave vectors parallel, $Q$, and perpendicular, $q$, to the filament:

$$
Q^{3}=q^{2} k_{B} T / \kappa
$$

Such relations, expressing the anisotropic scaling of lengths in two different directions, are most generally expressed in terms of roughness exponents, ${ }^{15} l \sim L^{5}$; for a semiflexible chain $\zeta=3 / 2$ as we have seen. It is this anomalous scaling of lengths parallel and perpendicular to the average axis of the filament which leads to the anomalous scaling seen in the experiments.

The dynamics of filament are described by the Langevin equation:

$$
\begin{aligned}
& \frac{\partial \mathbf{r}(s, t)}{\partial t}= \\
& \quad \int H\left(s, s^{\prime}\right)\left(-\kappa \nabla^{4} \mathbf{r}\left(s^{\prime}, t\right)+\sigma \nabla^{2} \mathbf{r}\left(s^{\prime}, t\right)+f\left(t, s^{\prime}\right)\right) \mathrm{d} s^{\prime}
\end{aligned}
$$

where $s$ and $s^{\prime}$ are two distinct points on the chain, $H\left(s, s^{\prime}\right)$ is the Oseen tensor describing the hydrodynamic interactions between monomers, and $f(t, s)$ is a random force due to collisions of the solvent molecules with the chain. The parameter $\sigma=\kappa / \xi^{2}$ is a Lagrange multiplier which ensures the correct crossover between the semiflexible and coil regime.

Because the wavelength of the light used in these experiments, $\lambda=0.514 \mu \mathrm{m}$, is small compared to the persistence length $\xi$ (about $6 \mu \mathrm{m}$ ), namely, $q \xi \gg 1$, the dynamic correlation function will be only sensitive to bending dynamic modes and our result is independent of $\sigma$; however, for technical reasons the calculation is simplified in the presence of the term in $\sigma$. From the Langevin equation (2.5) we find in this limit that

$$
i \omega=H(Q) \kappa Q^{4}
$$

where $\omega$ is the frequency and $Q$ the wave vector of a disturbance on the filament. The Oseen tensor, $H\left(s, s^{\prime}\right)$, decays as $1 /\left|s-s^{\prime}\right|$ for large separations; its Fourier transform thus varies as $\log (Q)$ for the length scale that we are interested in. If we now substitute relation 2.4 for $Q$, we find the scaling for the characteristic frequency $\Omega$ associated with transverse fluctuations with scattering vector $q$ is

$$
\Omega \sim q^{8 / 3} H\left(q^{2 / 3} \xi^{1 / 3}\right) \xi^{-1 / 3}
$$

Asymptotically, this frequency scales as $q^{8 / 3} \log (q d)$ (with $d$ a microscopic cutoff) and is the one of the main results of this paper. We note that in classical theories of polymer dynamics it is necessary to make the approximation of pre-averaging the Oseen tensor. Here, for length scales small compared with the persistence length, the relative fluctuations in the position of monomers are small; the pre-averaging of the tensor should be exact. ${ }^{16}$

It should be noted that increasing the rigidity of the filament slows the dynamics since to produce a given transverse fluctuation is necessary to excite longer wavelength excitations. In the next section we shall give a more detailed derivation of these results together with a calculation of the line shape measured in a scattering experiment. Again, we should note that this result is only valid in the limit $q \xi \gg 1$; for small $q \xi$ we expect to cross over to the conventional Rouse and Zimm regimes. We also note that this result is only expected to be valid in concentration ranges where $q^{-1}$ and $\xi^{1 / 3} q^{-2 / 3}$ are small compared with the mesh size formed by the actin filaments; in higher concentration ranges the behavior becomes more complicated and is discussed in section IV.

\section{Dynamic Structure Factor}

The energy of an excitation is quadratic in the coordinates $\mathbf{r}$, which lets us write immediately

$$
g(q, t)=\int \exp \left(-\frac{1}{2} q^{2}\left\langle(z(s, t)-z(0,0))^{2}\right\rangle\right) \mathrm{d} s
$$

where we have replaced the discrete sum of eq 2.1 by an integral equivalent.

The correlation function in the exponential is calculated from the Langevin equation for a semiflexible chain (2.5), separating the correlation function into static and dynamic parts:

$$
\left\langle\left(z(s, 0)-z\left(s^{\prime}, 0\right)\right)^{2}\right\rangle+2\left\langle\left(z(s, 0) z\left(s^{\prime}, 0\right)-z(s, 0) z\left(s^{\prime}, t\right)\right)\right\rangle
$$

We will calculate separately those two different contributions to the correlation function in the following.

First, the static correlation function is found from the following differential equation:

$$
\left(\kappa \nabla^{4}-\sigma \nabla^{2}\right) \mathbf{G}_{0}\left(s ; s^{\prime}, s^{\prime \prime}\right)=k_{B} T\left(\delta\left(s-s^{\prime}\right)-\delta\left(s-s^{\prime \prime}\right)\right)
$$

with boundary conditions

$$
\left(\kappa \nabla^{3}-\sigma \nabla\right) \mathbf{G}_{0}=0 ; \nabla^{2} \mathbf{G}_{0}=0
$$

for $x=0$ and $x=L$. The desired static part of eq 3.2 is simply given by $\mathbf{G}_{0}\left(s ; s, s^{\prime}\right)-\mathbf{G}_{0}\left(s^{\prime} ; s, s^{\prime}\right)$. Taking the limit of large $L$ and expanding in the limit $\left|s^{\prime}-s\right| \xi^{-1} \ll 1$, we find

$$
\left\langle\left(z\left(s^{\prime}, 0\right)-z(s, 0)\right)^{2}\right\rangle \approx \frac{k_{B} T}{\kappa}\left(-\frac{\left|s-s^{\prime}\right|^{3}}{12}+\frac{\xi}{2}\left(s-s^{\prime}\right)^{2}\right)
$$

The dynamic correlation function can be found by solving for the Green function associated with the Langevin equation (2.5). Equipartition is used to calculate the spacial correlation function $\left\langle\mathbf{f}(q) \cdot \mathbf{f}\left(q^{\prime}\right)\right\rangle$ for the stochastic force. For the time scales that we are interested in, the dynamics of the chain are dominated by bending elasticity. In this limit $\sigma p^{2}$ is negligible compared to $\kappa p^{4}$, and we find that

$$
\begin{aligned}
& \left\langle\left(z(s, 0) z\left(s^{\prime}, 0\right)-z(s, 0) z\left(s^{\prime}, t\right)\right)\right\rangle= \\
& \quad \frac{1}{2 \pi} \frac{k_{B} T}{\kappa} \int \frac{\exp \left(i p\left(s-s^{\prime}\right)\right)}{p^{4}}\left(1-\exp \left(-\kappa p^{4} H(p) t\right)\right) \mathrm{d} p
\end{aligned}
$$
form

The Oseen tensor $H\left(s, s^{\prime}\right)$ for a rod has the approximate

$$
H\left(s, s^{\prime}\right)=\frac{1}{8 \pi \eta} \frac{1}{a+\left|s-s^{\prime}\right|}
$$

valid for $\left|s-s^{\prime}\right|$ large compared with $a$, a microscopic cutoff 


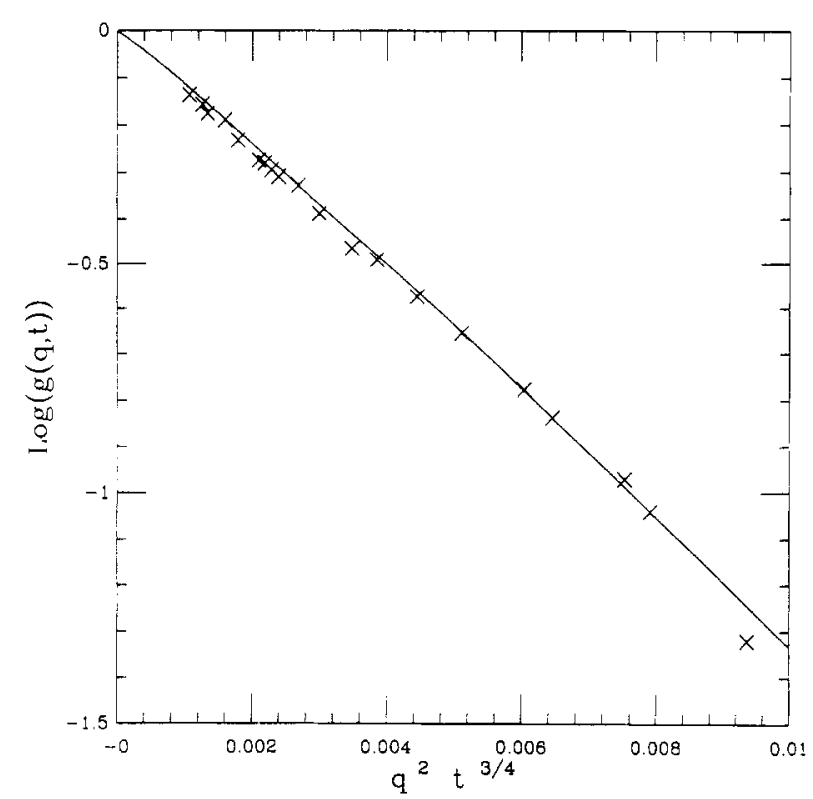

Figure 1. Plot of scaling function $g(q, t)$ on a logarithmic scale as a function of $q^{2} t^{3 / 4}$, from the paper of Piekenbrock and Sackmann $n^{13}$ at scattering angle of $\pi / 2$. The concentration of actin is $0.04 \mathrm{mg} / \mathrm{mL}$. The continuous curve is a fit with eq 3.9 with a mean slope of 129 (cgs units); see text. Curvature due to logarithmic corrections is small.

comparable to the size of a monomer. This function can be Fourier transformed ${ }^{17}$ to give

$$
H(p)=\left(-\gamma-\log (p a)+O(p a)^{2}\right) / 4 \pi \eta a
$$

where $\gamma \approx 0.57$.

From the static and dynamic contributions (3.5) and (3.6) of the correlation function we finally find for the dynamic structure factor:

$$
\mathbf{g}(q, t) \approx \int \exp \left(-\frac{q^{2}}{6} s^{2}-\frac{k_{B} T}{\kappa} q^{2}(\kappa t)^{3 / 4} \mathbf{F}(s, t)\right) \mathrm{d} s
$$

with

$$
\mathbf{F}(s, t)=\frac{1}{2 \pi} \int \exp \left(\frac{i s}{(\kappa t)^{1 / 4}} y\right) \frac{1-\exp \left(y^{4} H\left(y /(\kappa t)^{1 / 4}\right)\right)}{y^{4}} \mathrm{~d} y
$$

The function $\mathbf{F}(s, t)$ characterizes the propagation of a disturbance due to bending modes on a filament. The width of a wave packet grows subdiffusively with the law $s \sim t^{1 / 4}$, which is associated with the dispersion relation $\omega \sim q^{4}$. Rather surprisingly, the static contribution to the integral is dominated by the analytic term $\left(s-s^{\prime}\right)^{2} \xi / 2$ rather than the nonanalytic contribution $\left|s-s^{\prime}\right|^{3} / 12$ in eq 3.5. This means that $\mathbf{g}(q, t)$ is not a scaling function of its arguments. However, substitution of typical numerical values for all the constants shows that except at very small scattering angles we can replace function $\mathbf{F}(s, t)$ by $\mathbf{F}(0, t)$ since the factor $\exp \left(-q^{2} s^{2} / 6\right)$ in eq 3.9 cuts off the integral in $s$ extremely rapidly. Thus

$$
\mathbf{g}(q, t) \approx \mathbf{g}(q, 0) \exp \left(-\frac{k_{B} T}{\kappa} \mathbf{F}(0, t) q^{2}(\kappa t)^{3 / 4}\right)
$$

The function $\mathbf{F}(0, t)$ has been calculated numerically. If we take a typical value of $6 \mu \mathrm{m}$ for the persistence length, 8,9 the coefficient of $t^{3 / 4}$ in the exponential of eq 3.11 is 120 (cgs units) for a scattering angle of $\pi / 2$ for times of the order of $10^{-3} \mathrm{~s}$. We have replotted the data of refs 13 in Figure 1 and find a slope of $129 \pm 10$ (cgs units). Other scattering angles give some $30 \%$ variation in the slope, so we can consider this as an excellent agreement. Over the range of times and wave vectors used in these experiments the variation of the logarithmic correction in $F(0, t)$ is small; thus the decay is close to the law $\exp \left(-A q^{2} t^{3 / 4}\right)$; however, its presence is crucial to obtain quantitative agreement for the coefficient $A$. We note that to a very good approximation the slope $A$, in a plot of $\log (\mathbf{g}(q, t))$ as a function of $t^{3 / 4}$, varies as $\xi^{1 / 4}$.

\section{Semidilute Regime}

Until now we have not considered the interactions between filaments. For long times (of the order of $100 \mathrm{~ms}$ in the experiments of ref 13) and high concentrations, interactions between the filaments become important. Experimentally, this manifests itself by a decay of $g(q, t)$ which is estimated to be $\exp \left(-q^{2} t^{\alpha}\right)$ with $1 / 3>\alpha>1 / 7$. Theoretically, we can expect interactions between filaments to be important when the longest length scale in the single filament problem becomes comparable with the distance between filaments $\Lambda$. Thus we argue from eq 2.4 that for the above picture of independent filaments to hold we require that $\xi q^{-2 / 3} \ll \Lambda$. At this moment direct contacts between the filaments are still rare, but the hydrodynamic coupling (via the Oseen tensor) becomes important.

We have seen that for a single filament the dynamic structure factor can be expressed as

$$
g(q, t) \sim \exp \left(-q^{2} \int_{0}^{t} G\left(t^{\prime}, 0\right) \mathrm{d} t^{\prime}\right)
$$

By making a simple scaling argument for the form of $G(x, t)$ in three dimensions, we shall now estimate the form of $g(q, t)$ in the case of interacting filaments.

$G(x, t)$ has the simple physical interpretation as the response of the system to an impulsion as the origin at time zero. In a system of randomly oriented fibers dominated by bending elasticity the propagation from the origin should obey the law $x \sim t^{1 / 4}$ found in section III. In our arguments we shall neglect all logarithmic corrections. There is an important assumption in this propagation law: that the hydrodynamic interaction which varies as $1 / r$ in a dilute solution is screened for large distances; otherwise, we would have instantaneous transmission of forces over macroscopically large distances. Thus the dynamics of points which are separated by a distance which is much larger that $\lambda$ is effectively decoupled. Invariance of the system by translation then implies the presence of a local conserved quantity (by Noethers theorem) which is equal to $\int G(t, x) \mathrm{d}^{3} x$. This conservation law has the simple interpretation as the conservation of the center of mass in the absence of external forces. The conservation law immediately implies the following scaling form for $G$ :

$$
G(t, x)=t^{-3 / 4} G\left(x / t^{1 / 4}\right)
$$

The equivalent statement for a single filament is that $G(x, t)$ $=t^{-1 / 4} G\left(x / t^{1 / 4}\right)$, which is easily verified to be true using the results of section III. Note that for the threedimensional form of this relation to hold, nearby filaments (separated by a distance of order $\lambda$ ) must be strongly coupled; however, as we have already noted, this is true because of the form of the Oseen tensor; if we consider a segment of filament with length equal to $\lambda$, the hydrodynamic coupling with a nearby segment is comparable in strength with the coupling of the segment with itself. Thus the disturbance does not propagate along a single filament but rather distributes itself among filaments on length scales larger than $\lambda$. Substituting eq 4.2 into eq 4.1 , we find that

$$
g(q, t) \sim \exp \left(-q^{2} t^{1 / 4}\right)
$$

which seems compatible with the experimental data. 


\section{Concluding Discussion}

We have shown that the scattering of a dilute solution of semiflexible chains can be understood using simple scaling arguments and that the characteristic frequencies scale as $\Omega \approx q^{8 / 3} \log (q)$. Our analysis shows that the results of dynamic light scattering experiments are consistent with earlier, more intrusive, microscopy methods. We conclude that there does not seem to be a substantial variation of the flexibility of actin filaments in the presence of phallodin dyes, unlike suggestions in ref 13 .

We note that all those experiments are performed in the presence of magnesium or calcium ions, which are necessary for the polymerization of actin filaments from globular monomers. ${ }^{13}$ Without these ions, the actin molecule is fragile and easily denatured. The presence of these ions in solution can change the persistence length, first by screening electrostatic interactions between ions on the filament and second by incorporation in the interior of the protein. We have calculated ${ }^{18}$ the electrostatic contribution to the persistence length of an actin filament and find a correction of only a few angstroms: direct electrostatic effects due to the presence of charges along the chain have little influence on the persistence length. The problem of incorporation of ions into a protein is much more difficult to treat theoretically. Replacing magnesium by other divalent ions such as calcium ${ }^{8}$ or chromium ${ }^{19}$ could lead to conformation changes within, or on the surface of, one of the globular proteins. Thus we might expect that the exact mechanical constants of a filament are quite sensitive to the physical chemistry of the buffer solution. Further experimentation is necessary to elucidate this point.

It would be interesting to try and study other physical systems in the regime $\xi q \gg 1$. It would seem to be difficult to find other systems which could be studied with optical methods; other biological filaments such as microtubules are perhaps too rigid. More promising is perhaps neutron scattering where a persistence length of $10 \mathrm{~nm}$ should give comparable effects to those discussed here. In such systems the curve of $\mathbf{g}(q, t)$ as a function of $q^{2} t^{3 / 4}$ plotted in Figure 1 should be recalculated; we would expect that the variation in the curvature due logarithmic corrections will be more important on this length scale.

Acknowledgment. We wish to thank E. Sackmann of introducing us to this problem. We also wish to thank M.-F. Carlier, J. Candau, J.-P. Carton, L. Leibler, S. Leibler, D. Pantaloni, and J. Prost for many helpful discussions. E.F. is a scientist of the contingent supported by the DRET.

\section{References and Notes}

(1) Dubois-Violette, E.; de Gennes, P. G. Physics 1967, 3, 181.

(2) Adam, M.; Delsanti, M. Macromolecules 1977, 10, 1229.

(3) Doi, M.; Edwards, S. F. The Theory of Polymer Dynamics; Oxford Science Publication: Oxford, 1986. De Gennes, P.-G. Scaling Concepts in Polymer Physics; Cornell University Press: Ithaca, 1979.

(4) Darnel, J.; Lodish, H.; Baltimore, D. Molecular Cell Biology; Scientific American Books: 1986.

(5) Takebayashi, T.; Morita, Y.;Oosawa, F. Biochim. Biophys. Acta $1977,492,357$.

(6) Korn, E. D.; Carlier, M.-F.; Pantaloni, D. Science 1987, 238, 638.

(7) Yanagida, T.; Nakase, M.; Nishiyama, K.; Oosawa, F. Nature $1984,307,58$

(8) Oosawa, F. Biophys. Chem. 1980, 11, 443.

(9) Fujime, S. Adv. Biophys. 1972, 3, 1.

(10) Schmidt, C. F.; Barmann, M.; Isenberg, G.; Sackmann, E. Macromolecules 1989, 22, 3638.

(11) Mueller, O.; Gaub, H. E.; Barmann, M.; Sackmann, E. Macromolecules 1991, 24, 3111 .

(12) Janmey, P. A. Curr. Opin. Cell Biol. 1991, 2, 11.

(13) Piekenbrock, Th.; Sackmann, E. Biopolymers 1992, 32, 1471. Piekenbrock, Th. Thesis of the Technical University of Munich, Department of Physics, 1991.

(14) Harris, R. A.; Hearst, J. E. J. Chem. Phys. 1966, 44, 2595.

(15) Fisher, M. I. J. Stat. Phys. 1984, 34, 667.

(16) Allegra, G.; Higgins, J. S.; Ganazzoli, F.; Luccelli, E.; Bruckner, S. Macromolecules 1984, 17, 1253.

(17) Abromowitz, M.; Stegun, I. Handbook of Mathematical Functions; Dover: New York, 1972; p 232.

(18) Skolnick, J.; Fixman, M. Macromolecules 1977, 10, 944.

(19) Valentin-Ranc, C.; Carlier, M.-F. J. Biol.Chem. 1989, 35, 20871. 